\title{
Recognition of pneumococcal isolates by antisera raised against PspA fragments from different clades
}

\begin{abstract}
Correspondence
Eliane N. Miyaji

enmiyaji@butantan.gov.br
\end{abstract}

Received 28 September 2007

Accepted 7 November 2007

\author{
Michelle Darrieux, ${ }^{1} \dagger$ Adriana T. Moreno, ${ }^{1} \dagger$ Daniela M. Ferreira, ${ }^{1}$ \\ Fabiana C. Pimenta, ${ }^{2}$ Ana Lúcia S. S. de Andrade, ${ }^{2}$ Alexandre P. Y. Lopes, ${ }^{1}$ \\ Luciana C. C. Leite ${ }^{1}$ and Eliane N. Miyaji ${ }^{1}$ \\ ${ }^{1}$ Centro de Biotecnologia, Instituto Butantan, Av. Vital Brasil, 1500, 05509-900, São Paulo, SP, \\ Brazil \\ ${ }^{2}$ Instituto de Patologia Tropical e Saude Publica, Universidade Federal de Goias, Goiania, Brazil
}

\section{INTRODUCTION}

Streptococcus pneumoniae is responsible for millions of deaths worldwide every year (Cherian, 2007). The increase in antibiotic resistance, together with the high cost and limited coverage of the current conjugate vaccine, reinforce the need for cost-effective strategies. Recent reports on strain replacement in pneumococcal disease further support the necessity of developing alternative vaccines (Hicks et al., 2007; Singleton et al., 2007). Several proteins have been investigated as vaccine candidates against $S$. pneumoniae infection, including pneumococcal surface protein A (PspA), an immunogenic virulence factor (Tai, 2006) that is able to elicit high antibody levels in humans (Nabors et al., 2000) and to protect mice passively against challenge with virulent pneumococcal strains (Briles et al., 2000).

PspA has five domains: (i) a signal peptide, (ii) an $\alpha$-helical and charged $\mathrm{N}$-terminal domain, (iii) a proline-rich region, (iv) a choline-binding domain and (v) a short hydrophobic tail (Yother \& Briles, 1992; Yother \& White, 1994) (Fig. 1).

†Both authors contributed equally to this work.

Abbreviation: CDR, clade-defining region.
The N-terminal moiety is a well-exposed part of the molecule (Daniels et al., 2006; McDaniel et al., 1994), and also its functional fragment, interacting with the immune system (Shaper et al., 2004).

The N-terminus of PspA exhibits serological variability and can be divided into three regions, A, B and C. Region A is followed by an even more divergent region of about 100 aa, region B (McDaniel et al., 1994). This $\mathrm{N}$-terminal region is followed by a proline-rich region, know as $\mathrm{C}$. The $\mathrm{B}$ region formed the basis for the classification of PspA into six clades and three families, and is thus also referred to as the clade-defining region (CDR) (Hollingshead et al., 2000). Surveillance studies have shown that PspA families 1 and 2 are present in at least $90 \%$ of isolates, with prevalence rates of about $50 \%$ each (Beall et al., 2000; Brandileone et al., 2004; Heeg et al., 2007; Hollingshead et al., 2006; Ito et al., 2007; Mollerach et al., 2004; Payne et al., 2005; Pimenta et al., 2006; Sadowy et al., 2006; Vela Coral et al., 2001). Previous studies have shown that the level of crossreactivity among different PspAs roughly follows the degree of similarity among the amino acid sequences, with a tendency for a higher cross-reactivity within the same family (Nabors et al., 2000). This suggests that a 


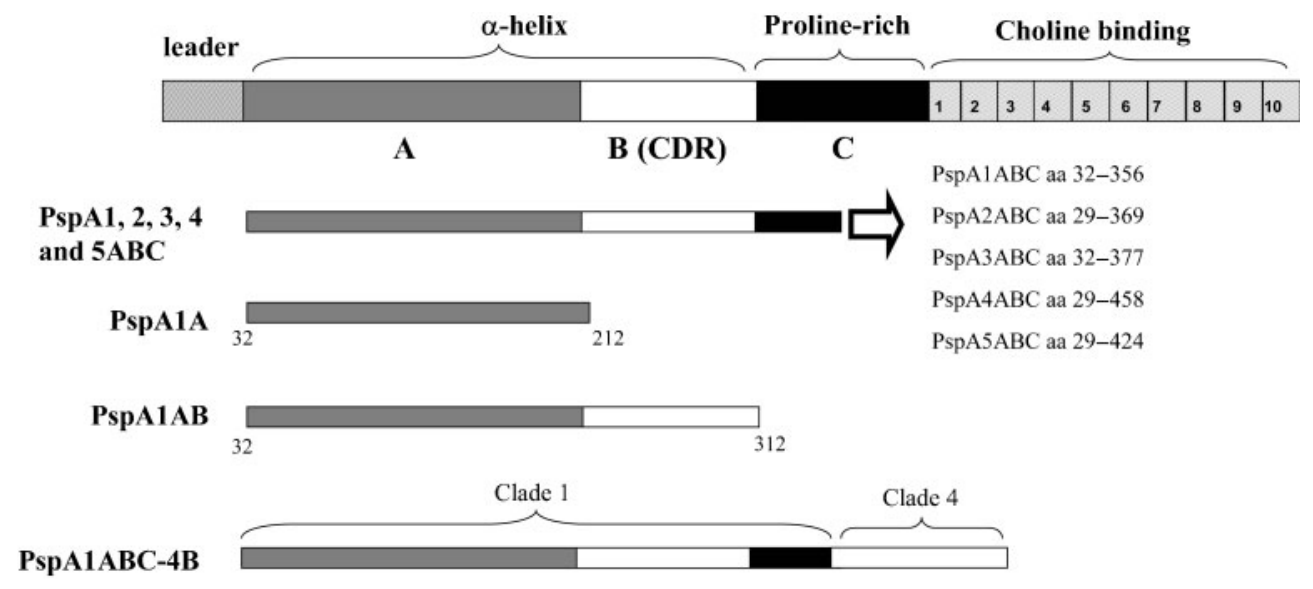

Fig. 1. Schematic diagram of PspA fragments obtained in this study. At the top is the whole PspA molecule, containing the Nterminal $\alpha$-helical domain (including regions $A$ and $B$ ), the proline-rich region $(C)$ and the choline-binding domain. The recombinant fragments are represented showing the distinct domains. The hybrid PspA1ABC-4B contained the N-terminal domain plus the proline-rich region of a PspA clade 1 fused to the CDR (B region) of a clade 4 PspA. The initial and final amino acids are shown for each fragment.

PspA-based vaccine should contain at least one fragment from each major family (family 1 , comprising clades 1 and 2 , and family 2, comprising clades 3,4 and 5) (Hollingshead et al., 2000). To investigate the level of cross-reactivity within PspA families and clades, we analysed the recognition of a panel of 35 pneumococcal isolates collected during a surveillance study in Brazil (Pimenta et al., 2006) by antisera raised against PspA fragments from clades 1 to 5 . We also investigated the contribution of different regions of the $\mathrm{N}$-terminus of PspA to its immunogenicity in order to elucidate the best composition for a PspA-based anti-pneumococcal vaccine.

\section{METHODS}

Construction of PspA fragments and hybrids. All cloning procedures were performed in Escherichia coli $\mathrm{DH} 5 \alpha$ grown in Luria-Bertani medium supplemented with ampicillin $\left(100 \mu \mathrm{g} \mathrm{ml}^{-1}\right)$. DNA fragments encoding the $\mathrm{N}$-terminal region of PspA clades 1 to 5 (A, B and C regions) were amplified by PCR from the genomic DNA of pneumococcal strains 435/96, 371/00, 259/98, 255/00 and 122/02, respectively (GenBank accession nos AY082387, EF649968, AY082389, EF649969 and EF649970). The forward (F) and reverse (R) primers used for amplification of the PspA fragments were: $5^{\prime}$ CTCGAGGAAGAAGCGCCCGTAGC-3' (PspA1F and PspA3F); 5' TAGTtatctagatgGtTGtgGtGCTGAAG-3' (PspA1R, PspA3R

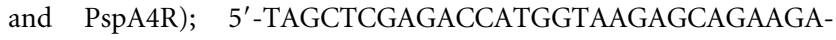
AGCC-3' (PspA2F, PspA4F and PspA5F); 5'-AGTTATCTAGATTTTGGTGCAGGAGCTGG-3' (PspA2R and PspA5R). The gene products were cloned into a pGEMT-Easy vector (Promega) and the correct sequences were confirmed by DNA sequencing. The pGEMTEasy/pspA constructs were digested with the appropriate restriction endonucleases and the resulting fragments were subcloned into linearized pAE- $6 \times$ His vector (Ramos et al., 2004). The hybrid PspA1ABC-4B (Miyaji et al., 2002) and its expression vector (Darrieux et al., 2007) have been described previously. Fig. 1 shows a scheme of the resulting PspA fragments.
PspA expression and purification. The pAE- $6 \times$ His vector containing the $p s p A$ constructs was used to transform BL21(DE3)SI E. coli competent cells (Invitrogen). Protein expression was induced in mid-exponential-phase cultures by the addition of $300 \mathrm{mM} \mathrm{NaCl}$. The recombinant proteins, bearing an $\mathrm{N}$-terminal histidine tag, were purified from the soluble fraction by affinity chromatography using $\mathrm{Ni}^{2+}$-charged resin (HisTrap HP; GE Healthcare) in an AKTAprime apparatus (GE Healthcare). Elution was carried out with $250 \mathrm{mM}$ imidazole. The purified fractions were analysed by SDS-PAGE, dialysed against $10 \mathrm{mM}$ Tris/ $\mathrm{HCl}(\mathrm{pH} 8) / 20 \mathrm{mM} \mathrm{NaCl} / 0.1 \%$ glycine and stored at $-20{ }^{\circ} \mathrm{C}$.

Pneumococcal strains. All strains used in this study were maintained as frozen stocks $\left(-80{ }^{\circ} \mathrm{C}\right)$ in Todd-Hewitt broth supplemented with $0.5 \%$ yeast extract (THY) with $20 \%$ glycerol, and are listed in Table 1 . These strains have been classified previously by sequencing of the CDR (Pimenta et al., 2006).

Animals and immunization. Female $\mathrm{BALB} / \mathrm{c}$ mice from Instituto Butantan (São Paulo, Brazil) were immunized subcutaneously with $5 \mu$ g recombinant PspA1ABC, PspA2ABC, PspA3ABC, PspA4ABC, PspA5ABC or the hybrid PspA1ABC-4B in Ringer's lactate solution using $50 \mu \mathrm{g} \mathrm{Al}(\mathrm{OH})_{3}$ as an adjuvant (final volume of $100 \mu \mathrm{l}$ per mouse). Adjuvant alone was used as a control. The animals were given three doses of protein at 7-day intervals. Sera were collected from mice by retro-orbital bleeding 1 week after the first immunization (for ELISA) or 2 weeks after the third immunization (for Western blotting).

Western blot analysis of anti-PspA sera. Pneumococcal isolates were plated on blood agar, transferred to THY and grown to an $\mathrm{OD}_{600}$ of 0.6 . The cell pellets were lysed by sonication and the soluble protein extracts were separated by SDS-PAGE and transferred to nitrocellulose membranes. Pooled anti-PspA sera (six mice per group) made against the recombinant PspA fragments of clades 1 to 5 or the clade 1-4 hybrid were added at a dilution of $1: 1000$ (sera collected after the third immunization), followed by incubation with horseradish peroxidase-conjugated goat anti-mouse IgG (diluted 1:1000; Sigma). Detection was performed with an ECL kit (GE Healthcare). 
Table 1. PspA clades and serotypes of the pneumococcal strains used in this study

\begin{tabular}{|c|c|c|c|c|c|c|c|c|c|}
\hline \multicolumn{4}{|c|}{ Family 1} & \multicolumn{6}{|c|}{ Family 2} \\
\hline \multicolumn{2}{|c|}{ Clade 1} & \multicolumn{2}{|c|}{ Clade 2} & \multicolumn{2}{|c|}{ Clade 3} & \multicolumn{2}{|c|}{ Clade 4} & \multicolumn{2}{|c|}{ Clade 5} \\
\hline $435 / 96^{*}$ & 1 & $371 / 00^{\star}$ & $6 \mathrm{~B}$ & $259 / 98^{*}$ & 14 & $255 / 00^{*}$ & 14 & $122 / 02^{*}$ & $23 \mathrm{~F}$ \\
\hline M12 & $6 \mathrm{~B}$ & M8 & $6 \mathrm{~B}$ & M10 & 3 & M2 & $6 \mathrm{~B}$ & P255 & $6 \mathrm{~A}$ \\
\hline P195 & $18 \mathrm{~A}$ & P176 & $9 \mathrm{~N}$ & P148 & $23 \mathrm{~F}$ & P71 & $23 \mathrm{~F}$ & & \\
\hline P266 & $23 \mathrm{~F}$ & P247 & $6 \mathrm{~B}$ & P212 & $23 \mathrm{~F}$ & P101 & $19 \mathrm{~F}$ & & \\
\hline P468 & $6 \mathrm{~A}$ & P278 & 12 & P539 & $19 \mathrm{~F}$ & P198 & $18 \mathrm{~A}$ & & \\
\hline P1151 & $11 \mathrm{~A}$ & P339 & $6 \mathrm{~B}$ & P762 & 14 & P414 & $10 \mathrm{~A}$ & & \\
\hline P1161 & 14 & P755 & 14 & P783 & $19 \mathrm{~F}$ & P1149 & $19 \mathrm{~F}$ & & \\
\hline
\end{tabular}

${ }^{\star}$ Strains from which the PspA fragments were cloned.

\begin{abstract}
Analysis of serum reactivity with different PspA regions. Recognition of the different PspA regions by antibodies to the recombinant proteins was analysed by ELISA. PolySorp 96-well plates (Nunc) were coated with fragments containing PspA regions $A, A B$ or $\mathrm{ABC}$ from clade $1\left(1 \mu \mathrm{g} \mathrm{m} \mathrm{m}^{-1}\right)$, washed three times with PBS containing $0.1 \%$ Tween 20 and blocked with $10 \%$ non-fat dried milk in PBS. The plates were then incubated with serial dilutions of individual sera from mice immunized with PspA1ABC or PspA1ABC4B (sera collected after the first immunization) in $1 \%$ BSA in PBS at $37^{\circ} \mathrm{C}$ for $1 \mathrm{~h}$. The plates were washed again and incubated with horseradish peroxidase-conjugated goat anti-mouse IgG (diluted $1: 15$ 000; Sigma) in $1 \%$ BSA in PBS at $37{ }^{\circ} \mathrm{C}$ for $1 \mathrm{~h}$. Following three more washes, antibodies were detected by adding OPD substrate $[0.04 \% \quad o$-phenylenediamine in citrate/phosphate buffer ( $\mathrm{pH} 5)$ containing $0.01 \% \mathrm{H}_{2} \mathrm{O}_{2}$ ]. After colour development (10 $\mathrm{min}$ ), the reaction was stopped with $1.25 \mathrm{M} \mathrm{H}_{2} \mathrm{SO}_{4}$ and the $A_{492}$ was determined. The reciprocal titre was considered to be the inverse of the last dilution of serum that registered an $A_{492}$ of 0.10 (sera from mice immunized with adjuvant only yielded negligible absorbance). Differences between groups were analysed by Student's $t$-test.
\end{abstract}

\section{RESULTS AND DISCUSSION}

This work investigated the level of cross-reactivity within PspA clades and families using a panel of 35 pneumococcal isolates (eight PspA1 strains, eight PspA2, eight PspA3, eight PspA4 and three PspA5 strains) and polyclonal sera made against PspA fragments of the five prevalent clades (clades 1 to 5). Western blot analysis revealed considerable variation in the recognition of isolates by the antibodies to the different clades. In general, there was strong recognition of isolates containing PspAs of the same clade as that used for production of the sera, and recognition decreased at the same rate as the level of similarity between the clades (Fig. 2).

Anti-PspA1ABC antibodies were able to recognize PspA in all clade 1-containing strains and half of the clade 2containing strains tested, whilst no reactivity with family 2 (clades 3-5) was observed (Fig. 2a). Anti-PspA2ABC antibodies revealed the narrowest recognition capacity, with positive reactions seen with only half of the PspA clade 2-containing strains, two clade 1 isolates and none of the other PspA clades (Fig. 2b). Anti-PspA3ABC antibodies were strongly reactive with all clade 3 - and two clade 2containing isolates; interestingly, there was no reactivity with the other PspA clades of family 2 (clades 4 and 5) (Fig. 2c). Anti-PspA4ABC and anti-PspA5ABC antibodies showed the broadest recognition capacity; anti-PspA4ABC reacted with all but four pneumococcal extracts (P174 and P278 from clade 2 and M10 and P5 from clade 3; Fig. 2d), whilst anti-PspA5ABC antibodies recognized six clade 1 , four clade 2, all clade 3 and 5, and six clade 4 PspAcontaining strains (Fig. 2e). These results showed that, among the PspA fragments analysed, PspA4ABC would be the most suitable for the induction of broad reactivity and that the inclusion of one fragment from family 1 (PspA1ABC) and another from family 2 (PspA4ABC) would be an even more effective strategy. Furthermore, the low reactivity shown by the anti-PspA2ABC antiserum indicated that the inclusion of more representative sequences is also an important issue, as sequence analysis has shown that the PspA clade 2 fragment used in this work is more divergent from other clade 2 PspAs sequenced by Hollingshead et al. (2000) compared with the clade 1, 3, 4 and 5 fragments. Another relevant aspect could be the fragment size: whilst the smaller fragments (from PspA clades 1, 2 and 3) induced antibodies with reduced crossreactivity, the longer fragments (from PspA clades 4 and 5) had a much broader recognition.

Previous studies using monoclonal antibodies (mAbs) generated by immunization with a pool of heat-killed pneumococci have revealed that a combination of only two Abs was able to detect virtually all clinical isolates tested in the study, one of them being reactive with PspA family 1 strains and the other with family 2 strains (Kolberg et al., 2001). This reinforces the hypothesis that a PspA-based vaccine containing a single fragment from each major family should be effective against virtually all pneumococci. 


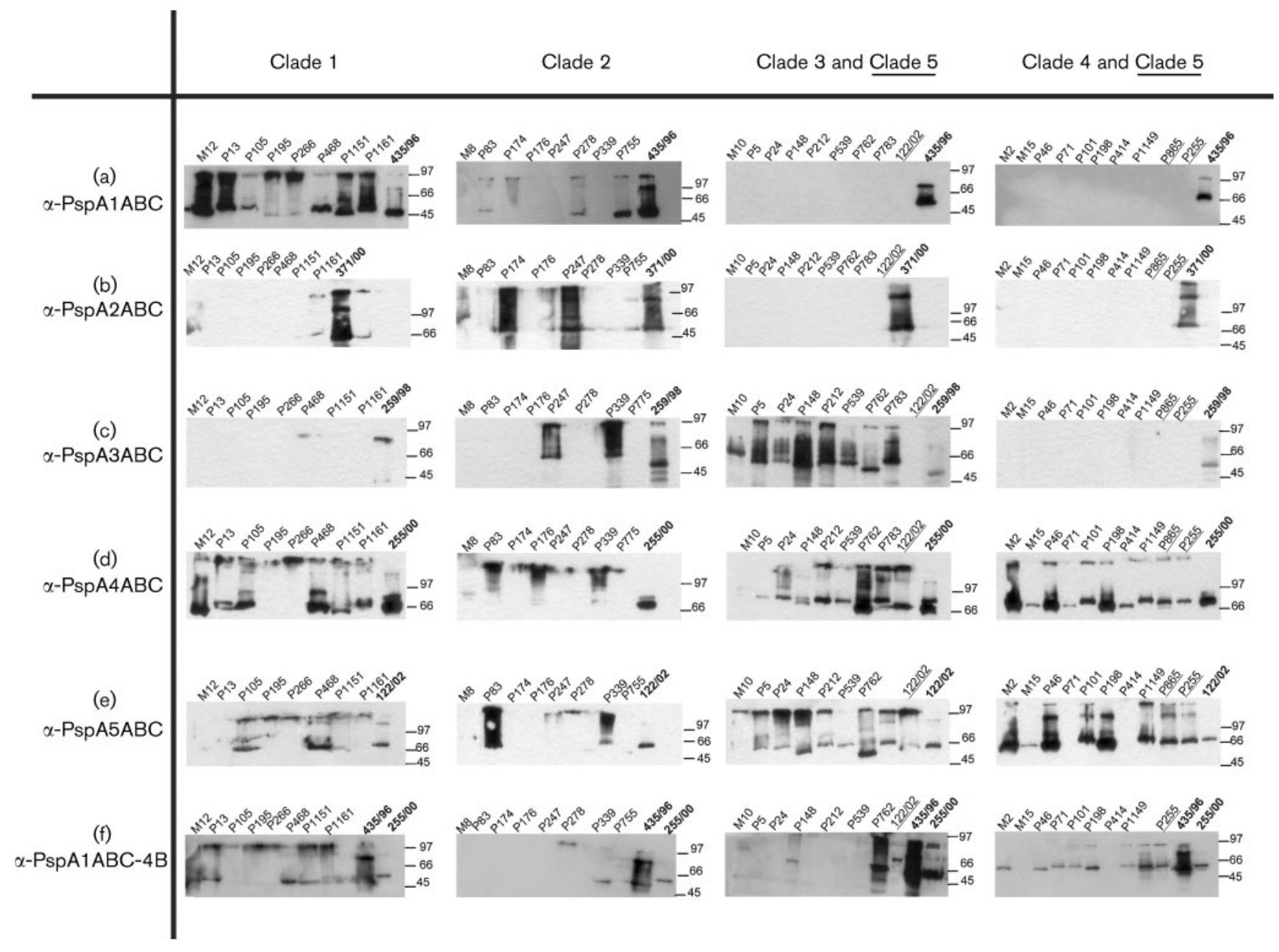

Fig. 2. Western blot analysis of the reactivity of anti-PspA antibodies within clades and families. Polyclonal sera from mice immunized with the $\mathrm{N}$-terminal regions of PspA clades 1 to 5 or the hybrid PspA1ABC-4B were tested for their reactivity with 35 pneumococcal extracts containing PspA from clades 1 to 5. Identification of the isolates is indicated on top of each blot; protein extracts of the strains from which the $p s p A$ fragments were cloned are indicated in bold and were used as positive controls; PspA clade 5-containing strains are underlined. Molecular mass markers $(\mathrm{kDa})$ are indicated on the right.

To test this hypothesis, we examined the reactivity pattern of sera made against the hybrid PspA1ABC-4B. The results indicated that antisera to this hybrid extended to a certain degree the recognition capacity of antibodies raised only against PspA1ABC, showing a strong reaction with PspA clade 1-, 4- and 5-bearing pneumococcal isolates, whilst cross-reactivity with clade 2 - and 3 -containing strains was much lower (only four out of 16 strains of these two clades were reactive) (Fig. 2f). In general, these results showed that the fusion is indeed able to enhance the level of crossreactivity, but that in order to achieve a broader effect, a longer fragment from family 2 should be included. In accordance with these results, our group has demonstrated that immunization with PspA hybrids induced antibodies with increased cross-reactivity, with the strongest effect obtained with the hybrid containing the longest PspA fragments (Darrieux et al., 2007).
Nabors et al. (2000) previously assessed the degree of crossreactivity of rabbit anti-PspA antibodies raised against PspAs from clades 1 to 5 against the same recombinant fragments. Analysis of PspA from family 1 showed that clades 1 and 2 had considerable cross-reactivity. However, in family 2, clade 3 PspA elicited antibodies with poor reactivity with clades 4 and 5 . Furthermore, anti-PspA2 human antibodies were also analysed for reactivity against pneumococcal strains bearing different PspAs by ELISA. There was an increase in titres against all strains tested, but these results were influenced by prior natural exposure of the immunized subjects to pneumococci. However, analysis of sera raised by PspAs from clades 1 to 5 against several strains bearing PspAs from the same clade was not performed.

As important as defining which PspA clade(s) induces the most cross-reactive antibodies is determination of the 
contribution of each protein domain to its immunogenicity; the two results combined could provide valuable information on the best composition for a PspA-based anti-pneumococcal vaccine.

It has been shown that immunization of mice with the A or $\mathrm{B}$ region induced high antibody levels against the whole $\mathrm{N}$ terminal fragment of a homologous fragment (Roche et al., 2003). In the present study, we proposed the opposite approach: identifying the level of recognition of each major domain by antibodies to the whole N-terminus.

Sera from mice immunized with PspA1ABC or the hybrid PspA1ABC-4B were analysed by ELISA against recombinant PspA clade 1 fragments containing regions $A, A B$ or $A B C$. Anti-PspA1ABC had a significantly higher reactivity with the longer fragments $(\mathrm{AB}$ and $\mathrm{ABC})$ compared with the smaller fragment (A) $(P=0.026)$. No significant differences were observed between $A B$ and $A B C$, indicating that the proline-rich region may have little contribution towards PspA1ABC immunogenicity in this model (Fig. 3a). The results also suggested that regions A and B were equally immunogenic. This result corroborates mapping studies using mAbs, which have shown that, from a pool of nine $\mathrm{mAbs}$, five were reactive against the $\mathrm{A}$ region and four against the $\mathrm{B}$ region, indicating that these

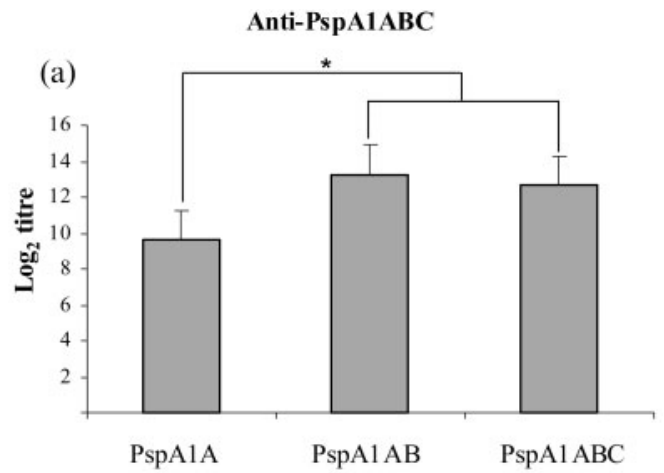

(b)

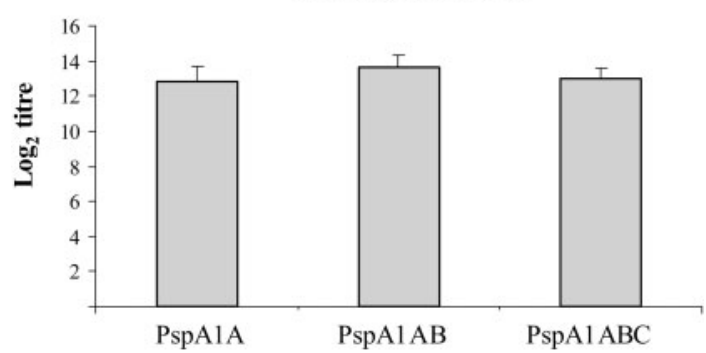

Fig. 3. Serum reactivity with different PspA regions. Polyclonal sera from mice immunized with recombinant PspA1ABC (a) or PspA1ABC-4B (b) were analysed by ELISA against recombinant PspA fragments containing region $A, A B$ or $A B C$ of clade 1 . The results are shown as $\log _{2}$ of the titre. ${ }^{*}$ Statistically significant difference $(P=0.026)$. two domains may be important for the immunogenicity of PspA (McDaniel et al., 1994). This recognition pattern is probably due to PspA folding back on itself to generate a coiled-coil structure, which allows these different domains to be exposed on opposite sides of the same coiled-coil unit (Jedrzejas et al., 2000; Senkovich et al., 2007). Moreover, a study with PspA fragments including different portions of the $\alpha$-helical domain has demonstrated that, for family 2 strains, fragments including both ends of the $\alpha$-helical region elicited the best protection (Roche et al., 2003). This result suggests that the $\mathrm{A}$ and $\mathrm{B}$ regions are important not only for the immunogenicity of PspA, but also for protection against pneumococcal challenge.

In contrast to what was observed with sera from the PspA fragment, antibodies made against the hybrid PspA1ABC4B did not show a significant difference in reaction with the A region compared with $\mathrm{AB}$ (Fig. $3 \mathrm{~b}$ ). This result may be due to differences in the hybrid structure, altering the accessibility of the regions to the immune system.

In conclusion, whilst it is important to determine which PspA fragments show the broadest cross-reactivity within the same family (or even the same clade, as is the case for the PspA clade 2 fragment), in order to extend reactivity to heterologous PspA families, it is important to have longer fragments for immunization, including the more conserved proline-rich region. This domain may also play a significant role in maintaining certain structural features necessary for the induction of cross-reactive antibodies. Furthermore, the use of a fusion protein bearing fragments of both families was able to increase the cross-reactivity of antisera, indicating that this could be a promising immunization strategy. Taken together, our results suggest that the best composition for a PspA-based anti-pneumococcal vaccine should include the whole $\mathrm{N}$-terminal region of PspA clades 1 and 4, which could be expressed as a hybrid protein, as these are able to induce antibodies with a broad cross-reactivity. Moreover, future studies should also evaluate whether the protective ability of the antibodies correlates with the crossreactivity data presented in this work.

\section{ACKNOWLEDGEMENTS}

This work was supported by CNPq (grant MCT/CNPq/PADCT \# 620023/2004-0), FAPESP, Fundação Butantan, FUNDAP and the Millenium Institute-Gene Therapy Network (MCT-CNPq). A. L.S.S. A. (grant no. 308043/2004-9), E. N. M. (grant no. 301256/ 2005-5), F. C. P. (grant no. 301646/2006-6) and L.C.C. L. (grant no. 351133/1997-6) are recipients of fellowships from CNPq.

\section{REFERENCES}

Beall, B., Gherardi, G., Facklam, R. R. \& Hollingshead, S. K. (2000). Pneumococcal $p s p A$ sequence types of prevalent multiresistant pneumococcal strains in the United States and of internationally disseminated clones. J Clin Microbiol 38, 3663-3669.

Brandileone, M. C., Andrade, A. L., Teles, E. M., Zanella, R. C., Yara, T. I., Di Fabio, J. L. \& Hollingshead, S. K. (2004). Typing of 
pneumococcal surface protein A (PspA) in Streptococcus pneumoniae isolated during epidemiological surveillance in Brazil: towards novel pneumococcal protein vaccines. Vaccine 22, 3890-3896.

Briles, D. E., Hollingshead, S. K., King, J., Swift, A., Braun, P. A., Park, M. K., Ferguson, L. M., Nahm, M. H. \& Nabors, G. S. (2000). Immunization of humans with recombinant pneumococcal surface protein A ( $\mathrm{rPspA}$ ) elicits antibodies that passively protect mice from fatal infection with Streptococcus pneumoniae bearing heterologous PspA. J Infect Dis 182, 1694-1701.

Cherian, T. (2007). WHO expert consultation on serotype composition of pneumococcal conjugate vaccines for use in resource-poor developing countries, 26-27 October 2006, Geneva. Vaccine 25, 6557-6564.

Daniels, C. C., Briles, T. C., Mirza, S., Hakansson, A. P. \& Briles, D. E. (2006). Capsule does not block antibody binding to PspA, a surface virulence protein of Streptococcus pneumoniae. Microb Pathog 40, $228-233$.

Darrieux, M., Miyaji, E. N., Ferreira, D. M., Lopes, A. P. Y., Ren, B., Briles, D. E., Hollingshead, S. K. \& Leite, L. C. (2007). Fusion proteins containing family 1 and family 2 PspA fragments elicit protection against Streptococcus pneumoniae that correlates with antibodymediated enhancement of complement deposition. Infect Immun 75, 5930-5938.

Heeg, C., Franken, C., van der Linden, M., Al-Lahham, A. \& Reinert, R. R. (2007). Genetic diversity of pneumococcal surface protein A of Streptococcus pneumoniae meningitis in German children. Vaccine 25, 1030-1035.

Hicks, L. A., Harrison, L. H., Flannery, B., Hadler, J. L., Schaffner, W., Craig, A. S., Jackson, D., Thomas, A., Beall, B. \& other authors (2007). Incidence of pneumococcal disease due to non-pneumococcal conjugate vaccine (PCV7) serotypes in the United States during the era of widespread PCV7 vaccination, 1998-2004. J Infect Dis 196, 1346-1354.

Hollingshead, S. K., Becker, R. \& Briles, D. E. (2000). Diversity of PspA: mosaic genes and evidence for past recombination in Streptococcus pneumoniae. Infect Immun 68, 5889-5900.

Hollingshead, S. K., Baril, L., Ferro, S., King, J., Coan, P. \& Briles, D. E. (2006). Pneumococcal surface protein A (PspA) family distribution among clinical isolates from adults over 50 years of age collected in seven countries. J Med Microbiol 55, 215-221.

Ito, Y., Osawa, M., Isozumi, R., Imai, S., Ito, I., Hirai, T., Ishida, T., Ichiyama, S. \& Mishima, M. (2007). Pneumococcal surface protein A family types of Streptococcus pneumoniae from community-acquired pneumonia patients in Japan. Eur J Clin Microbiol Infect Dis 26, 739-742.

Jedrzejas, M. J., Hollingshead, S. K., Lebowitz, J., Chantalat, L., Briles, D. E. \& Lamani, E. (2000). Production and characterization of the functional fragment of pneumococcal surface protein A. Arch Biochem Biophys 373, 116-125.

Kolberg, J., Aase, A., Michaelsen, T. E. \& Rodal, G. (2001). Epitope analyses of pneumococcal surface protein A: a combination of two monoclonal antibodies detects $94 \%$ of clinical isolates. FEMS Immunol Med Microbiol 31, 175-180.

McDaniel, L. S., Ralph, B. A., McDaniel, D. O. \& Briles, D. E. (1994). Localization of protection-eliciting epitopes on PspA of Streptococcus pneumoniae between amino acid residues 192 and 260. Microb Pathog 17, 323-337.

Miyaji, E. N., Ferreira, D. M., Lopes, A. P., Brandileone, M. C., Dias, W. O. \& Leite, L. C. (2002). Analysis of serum cross-reactivity and cross-protection elicited by immunization with DNA vaccines against Streptococcus pneumoniae expressing PspA fragments from different clades. Infect Immun 70, 5086-5090.
Mollerach, M., Regueira, M., Bonofiglio, L., Callejo, R., Pace, J., Di Fabio, J. L., Hollingshead, S. \& Briles, D. (2004). Invasive Streptococcus pneumoniae isolates from Argentinian children: serotypes, families of pneumococcal surface protein A (PspA) and genetic diversity. Epidemiol Infect 132, 177-184.

Nabors, G. S., Braun, P. A., Herrmann, D. J., Heise, M. L., Pyle, D. J., Gravenstein, S., Schilling, M., Ferguson, L. M., Hollingshead, S. K. \& other authors (2000). Immunization of healthy adults with a single recombinant pneumococcal surface protein A (PspA) variant stimulates broadly cross-reactive antibodies to heterologous PspA molecules. Vaccine 18, 1743-1754.

Payne, D. B., Sun, A., Butler, J. C., Singh, S. P., Hollingshead, S. K. \& Briles, D. E. (2005). PspA family typing and PCR-based DNA fingerprinting with BOX A1R primer of pneumococci from the blood of patients in the USA with and without sickle cell disease. Epidemiol Infect 133, 173-178.

Pimenta, F. C., Ribeiro-Dias, F., Brandileone, M. C., Miyaji, E. N., Leite, L. C. \& Sgambatti de Andrade, A. L. (2006). Genetic diversity of PspA types among nasopharyngeal isolates collected during an ongoing surveillance study of children in Brazil. J Clin Microbiol 44, 2838-2843.

Ramos, C. R., Abreu, P. A., Nascimento, A. L. \& Ho, P. L. (2004). A high-copy T7 Escherichia coli expression vector for the production of recombinant proteins with a minimal $\mathrm{N}$-terminal His-tagged fusion peptide. Braz J Med Biol Res 37, 1103-1109.

Roche, H., Hakansson, A., Hollingshead, S. K. \& Briles, D. E. (2003). Regions of PspA/EF3296 best able to elicit protection against Streptococcus pneumoniae in a murine infection model. Infect Immun 71, 1033-1041.

Sadowy, E., Skoczynska, A., Fiett, J., Gniadkowski, M. \& Hryniewicz, W. (2006). Multilocus sequence types, serotypes, and variants of the surface antigen PspA in Streptococcus pneumoniae isolates from meningitis patients in Poland. Clin Vaccine Immunol 13, 139-144.

Senkovich, O., Cook, W. J., Mirza, S., Hollingshead, S. K., Protasevich, I. I., Briles, D. E. \& Chattopadhyay, D. (2007). Structure of a complex of human lactoferrin N-lobe with pneumococcal surface protein A provides insight into microbial defense mechanism. J Mol Biol 370, 701-713.

Shaper, M., Hollingshead, S. K., Williams, J. H., Jr \& Briles, D. E. (2004). PspA protects Streptococcus pneumoniae from killing by apolactoferrin and antibody to PspA enhances killing of pneumococci by apolactoferrin. Infect Immun 72, 5031-5040.

Singleton, R. J., Hennessy, T. W., Bulkow, L. R., Hammitt, L. L., Zulz, T., Hurlburt, D. A., Butler, J. C., Rudolph, K. \& Parkinson, A. (2007). Invasive pneumococcal disease caused by nonvaccine serotypes among Alaska Native children with high levels of 7 -valent pneumococcal conjugate vaccine coverage. JAMA 297, 1784-1792.

Tai, S. S. (2006). Streptococcus pneumoniae protein vaccine candidates: properties, activities and animal studies. Crit Rev Microbiol 32, 139-153.

Vela Coral, M. C., Fonseca, N., Castaneda, E., Di Fabio, J. L., Hollingshead, J. K. \& Briles, D. E. (2001). Pneumococcal surface protein A of invasive Streptococcus pneumoniae isolates from Colombian children. Emerg Infect Dis 7, 832-836.

Yother, J. \& Briles, D. E. (1992). Structural properties and evolutionary relationships of PspA, a surface protein of Streptococcus pneumoniae, as revealed by sequence analysis. J Bacteriol 174, 601-609.

Yother, J. \& White, J. M. (1994). Novel surface attachment mechanism of the Streptococcus pneumoniae protein PspA. J Bacteriol 176, 2976-2985. 